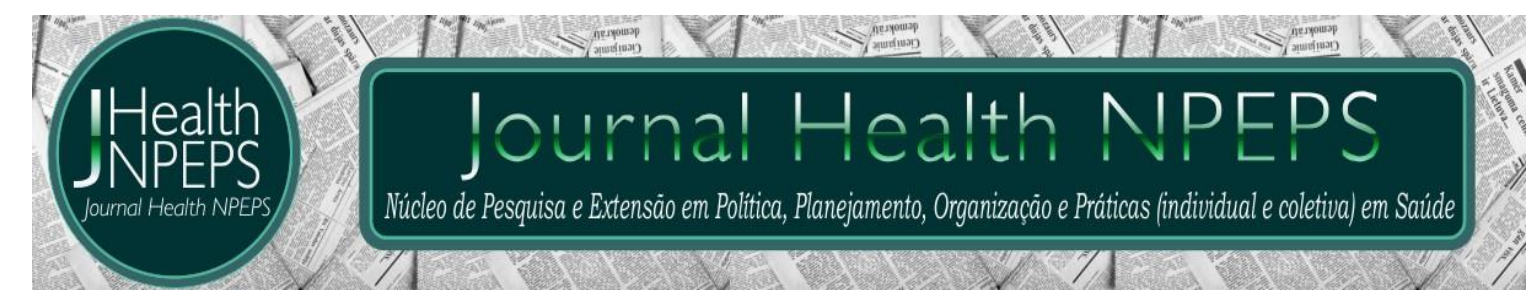

ARTIGO ORIGINAL

\title{
PERCEPÇÕES DOS PROFISSIONAIS DE ENFERMAGEM SOBRE O PACIENTE PÓS-TENTATIVA DE SUICÍDIO
}

NURSE PROFESSIONAL PERCEPTIONS TOWARDS TO POST SUICIDE ATTEMPT PATIENT

LAS PERCEPCIONES DE LOS PROFESIONALES DE ENFERMERÍA EN EL PACIENTE DESPUÉS DE INTENTO DE SUICIDIO

\begin{abstract}
Ykaro Hariel Alves de Oliveira Liba ${ }^{1}$, Alisséia Guimarães Lemes ${ }^{2}$, Pâmela Roberta de Oliveira $^{3}$, Vagner Ferreira do Nascimento ${ }^{4}$, Paula Isabella Marujo Nunes da Fonseca ${ }^{5}$, Rosa Jacinto Volpato6, Maria Aparecida Sousa Oliveira Almeida7, Tayane Próspero Cardoso ${ }^{8}$
\end{abstract}

\section{RESUMO}

Objetivo: identificar a percepção dos profissionais acerca dos cuidados prestados a pacientes que tentaram suicídio. Método: estudo descritivo, exploratório com abordagem qualitativa, realizado em um hospital público do interior mato-grossense, com 38 profissionais de enfermagem que atuam nos setores de emergência, clínica medica e UTI. A coleta de dados ocorreu nos meses de julho a setembro de 2014, por meio da aplicação de um questionário semiestruturado. A análise dos dados foi realizada por meio da Análise Temática. Resultados: a maioria dos profissionais são do gênero feminino, adultos jovens, com ensino médio completo, solteiros e católicos. Uma parte significativa dos entrevistados sentem-se capazes de prestar um suporte adequado as pessoas que tentaram suicídio e quanto as percepções dos profissionais em relação a estes pacientes variaram de indiferença à compreensão. Considerações finais: o estudo revelou que a equipe de enfermagem se mostrou capaz de prestar uma assistência a este perfil de paciente, por outro lado, demonstrou a necessidade de capacitações abordando aspectos que envolvem a saúde mental, podendo contribuir com sua formação e garantir uma assistência de qualidade aos pacientes.

Descritores: Cuidados de Enfermagem; Saúde Mental; Tentativa de Suicídio.

\footnotetext{
${ }^{1}$ Enfermeiro. Bacharel em Enfermagem. Universidade Federal de Mato Grosso (UFMT). Barra do Garças - MT - Brasil. Email: liboriobr@gmail.com

${ }^{2}$ Enfermeira. Doutoranda em Enfermagem Psiquiátrica (EERP/USP). Docente Assistente da Universidade Federal de Mato Grosso (UFMT). Barra do Garças - MT - Brasil. E-mail: alisseia@hotmail.com. Autor principal - Endereço para correspondência: Avenida Valdon Varjão, 6390. CEP 78600-000. Barra do Garças - MT- Brasil.

${ }^{3}$ Enfermeira. Mestre em Educação. Docente Assistente da Universidade Federal de Mato Grosso (UFMT). Barra do Garças - MT - Brasil. E-mail: pamela_veira@yahoo.com.br

${ }^{4}$ Enfermeiro. Doutorando em Bioética. Docente Assistente da Universidade do Estado de Mato Grosso (UNEMAT). Tangará da Serra - MT - Brasil. E-mail: vagnerschon@hotmail.com

${ }^{5}$ Enfermeira. Doutoranda em Ciências do Cuidado em Saúde. Universidade Federal Fluminense (UFF). Niterói - RJ Brasil. E-mail: paulaisabellafonseca@gmail.com

${ }^{6}$ Enfermeira. Mestranda em Enfermagem. Universidade Federal de São Carlos (UFSCar). São Carlos - SP - Brasil. Email: rosamjacinto@hotmail.com

7 Enfermeira. Bacharel em Enfermagem. Universidade Federal de Mato Grosso (UFMT). Barra do Garças - MT - Brasil. E-mail: almeidacida@hotmail.com

8 Enfermeira. Bacharel em Enfermagem. Universidade Federal de Mato Grosso (UFMT). Barra do Garças - MT - Brasil. E-mail: tayanepc@hotmail.com
} 


\begin{abstract}
Objective: to identify the perception of professionals about the care provided to patients who attempted suicide. Method: descriptive, exploratory qualitative study, conducted in a public hospital of Mato Grosso inside with 38 nursing professionals who work in emergency departments, medical clinic and UTI. Data collection occurred from July to September 2014, through the application of a semi-structured questionnaire. Data analysis was performed through thematic analysis. Results: most professionals are female, young adult, full and single high school. A significant proportion of respondents feel able to provide adequate support people who have attempted suicide and the perceptions of professionals for these patients ranged from indifference to understanding. Final considerations: the study showed that the nursing staff was capable of providing assistance to this patient profile, on the other hand, it demonstrated the need for training addressing issues surrounding mental health and may contribute to their training and ensure assistance quality to patients.

Descriptors: Nursing Care; Mental Health; Suicide, Attempted.
\end{abstract}

\title{
RESUMEN
}

Objetivo: identificar la percepción de los profesionales sobre la atención prestada a los pacientes que intentaron suicidarse. Método: estudio cualitativo exploratorio descriptivo, realizado en un hospital público de Mato Grosso en el interior con 38 profesionales de enfermería que trabajan en los servicios de urgencias, clínica médica y la UTI. Los datos fueron recolectados de julio a septiembre de 2014, mediante la aplicación de un cuestionario semiestructurado. El análisis de datos se realizó mediante el análisis temático. Resultados: la mayoría de los profesionales son mujeres, adulto joven, lleno y la escuela secundaria única. Una proporción significativa de los encuestados se siente capaz de proporcionar a las personas de apoyo adecuados que han intentado suicidarse y las percepciones de los profesionales de estos pacientes osciló entre indiferencia y la comprensión. Consideraciones finales: el estudio mostró que el personal de enfermería fue capaz de proporcionar asistencia a este perfil de paciente, por el contrario, que demostraron la necesidad de una formación abordar cuestiones relacionadas con la salud mental y puede contribuir a su formación y garantizar la asistencia calidad a los pacientes.

Descriptores: Atención de Enfermería; Salud Mental; Intento de Suicidio.

\section{INTRODUÇÃO}

O suicídio é o ato em que o indivíduo deliberadamente provoca sua própria morte. O comportamento suicida é toda ação que pode levar ao suicídio, desde o fato de se pensar e planejar passos (ideação), realizar o intento obtendo a falha (tentativa) ou o sucesso (suicídio) ${ }^{1}$.

Aproximadamente um milhão de pessoas morrem por suicídio em todo o mundo durante um ano. O Brasil, com coeficiente de mortalidade 4,5 de 100.000 habitantes em um ano (7,5 e 2 em homens e mulheres, respectivamente), relacionado com os coeficientes internacionais, considera-se baixo, porém, quando se observa a frequência, 
é elevada devido a sua dimensão populacional, atingindo o nono lugar na escala mundial ${ }^{2}$.

Os dados do Sistema de Informação sobre Mortalidade (SIM), apontam que em 2011, 9.852 suicídios foram registrados, correspondendo a aproximadamente 27 mortes por dia em todo o país, durante um $a^{3} o^{3}$. É reconhecido que a subnotificação de casos (associado ao despreparo dos profissionais de saúde) e suicídios camuflados em outros eventos (aparência homicida, acidentes) não permite a contabilidade real da quantidade de $\operatorname{casos}^{2,4}$.

Nesse cenário, Mato Grosso apresenta uma alta taxa de comportamentos suicidas, ocupando em 2014, o terceiro lugar de destaque em prevalência dessas ocorrências no centro oeste, perdendo apenas para o estado do Distrito Federal e Goiás ${ }^{5}$. Assim, o crescente aumento no número de casos de suicídio e tentativas de suicídio, que ceifam famílias e estruturas sociais aponta para urgência na intervenção dos profissionais de saúde, principalmente através de atividades de promoção da valorização da vida ${ }^{3}$.

E no universo dos profissionais de saúde, a equipe de enfermagem se distingui em recursos e possibilidades terapêuticas, quer seja em sua maior habilidade no exercício da comunicação, abordagem, diversidade teórica, construção facilitada de vínculos como na expertise na definição e execução da melhor estratégia de cuidado 4 .

Nesse sentido, os profissionais de enfermagem desempenham importante papel no atendimento preventivo e também pós tentativa de suicídio, pois o tempo destinado aos cuidados, podem propiciar espaço de escuta e presença terapêutica necessária nesse momento, levando a descoberta de potenciais ideias suicidas e diversidade de sofrimentos que podem encorajar um comportamento suicida ${ }^{3-4}$.

Diante disso, objetivou-se com esse estudo identificar as percepções dos profissionais de enfermagem acerca do atendimento prestados a pacientes que tentaram suicídio.

\section{MÉTODO}

Trata-se de um estudo descritivo exploratório e com abordagem qualitativa, desenvolvido com profissionais da equipe de enfermagem (Técnicos de enfermagem e enfermeiros) que atuam nos setores de emergência, clínica médica e Unidade de Terapia 
Intensiva (UTI) de um hospital público localizadas na região Médio Araguaia no interior de Mato Grosso.

Os critérios de inclusão dos participantes na pesquisa foram: profissionais com idade maior ou igual a 18 anos; ser enfermeiros ou técnicos de enfermagem que atuam nos setores de clínica médica, UTI e emergência; aceitar participar da pesquisa assinando o Termo de Consentimento Livre e Esclarecido (TCLE). Os critérios de exclusão foram: profissionais afastados do serviço por meio de atestado médico, licença maternidade, licença-prêmio ou férias no período da coleta de dados.

Para definição da amostra, utilizou-se uma amostragem não probabilística, por acessibilidade, perfazendo 54 profissionais, de ambos os gêneros. No entanto, só estiveram aptos a participar aqueles que atenderam aos critérios definidos, totalizando 38 profissionais, sendo oito enfermeiros e 30 técnicos em enfermagem.

A coleta de dados ocorreu nos meses de julho a setembro de 2014, por meio da aplicação de um questionário semiestruturado contendo questões abertas e fechadas, contemplando as características sociodemográficas (gênero, escolaridade, renda, estado civil e religião), o perfil profissional (função exercida na enfermagem, tempo de atuação na enfermagem e nos setores, histórico de suicídio na família e estudo da temática suicídio durante a formação profissional) e o lidar com o caso de tentativa do suicídio (enquanto profissional sente-se capaz de auxiliar um pacientes pós-tentativa de suicídio?; Você acredita que o suicídio está relacionado com alguma doença Mental?; Você consegue identificar fatores de risco no paciente que possa levá-lo à tentativa de suicídio no futuro ou agora?; enquanto profissional como você se sente durante uma abordagem ao paciente que tentou suicídio?; Qual o seu pensamento em relação aos pacientes que tentam suicídio, comparados aos pacientes internados que lutam pela vida? Você acredita que o indivíduo possa ter a autonomia de atentar contra a sua própria vida?; Na sua prática profissional, você saberia oferecer orientações para o indivíduo e seus familiares no pós-atendimento de maneira que eles possam buscar continuidade no atendimento relacionado à tentativa de suicídio?). Mediante aos dados coletados emergiram duas categorias neste estudo, sendo: o perfil da profissão e o enfrentamento ao caso de tentativa de suicídio.

A coleta de dados foi realizada nos setores de clínica médica, UTI e emergência nas salas de descanso (repouso) utilizada pela equipe de enfermagem de cada setor, visto que estas salas, apresentavam um ambiente calmo e silencioso essencial à 
aplicação do instrumento, posterior à assinatura do TCLE. Os questionários foram preenchidos pelos profissionais, não havendo interferência dos pesquisadores, a duração para o preenchimento do formulário foi em média de 30 minutos.

A análise dos dados foi realizada neste estudo por meio da Análise Temática, por comportar um feixe de relações e pode ser graficamente apresentada através de uma frase onde o tema é a unidade de significação que se liberta naturalmente de um texto analisado 6 .

Atendendo à garantia do sigilo de identidades dos participantes, utilizou-se para a apresentação dos relatos o uso da letra "E" para Enfermeiro e "T" para Técnico em Enfermagem, seguida pelo número arábico correspondente à ordem na entrevista em substituição aos próprios.

Todas as providências em relação à dimensão ética do estudo foram tomadas de acordo com a Resolução de ética 466/2012. O estudo foi aprovado pelo Comitê de Ética em Pesquisa da Universidade Federal de Mato Grosso/Campus Universitário do Araguaia, sob o protocolo número 515/705.

\section{RESULTADOS}

$\mathrm{Na}$ Tabela 1, encontram-se os dados sociodemográficos da população estudada, demonstrando que os profissionais se constituíram em sua maioria do gênero feminino, solteiros, com idade entre 21 a 60 anos, com ensino médio completo, renda mensal de 1 a 3 salários mínimos e católicos.

Quanto ao perfil da profissão, esses profissionais possuem mais de dez anos de profissão, atuando em média a cinco anos nessa instituição, 34\% no setor de UTI, 34\% na emergência e $32 \%$ na clínica médica.

Quanto ao histórico familiar de suicídio ou tentativa de suicídio entre os profissionais, os dados revelaram que $68 \%$ nunca vivenciaram essa situação, enquanto 29\% informaram episódio na família.

Quanto ao estudo da temática suicídio durante a formação profissional, 71\% dos profissionais afirmaram terem vistos, porém, apenas de forma superficial correspondendo apenas a um momento pontual e isolado dentro de uma determinada disciplina; $10 \%$ declararam ter tido um contato mais aprofundado considerado como uma aula, palestra ou minicurso e 16\% não tiveram qualquer instrução a esse respeito. 
Tabela 1 - Distribuição das variáveis sociodemográficas dos profissionais de enfermagem no interior do Médio Araguaia. Julho a setembro de 2014. Mato Grosso, Brasil $(n=38)$.

\begin{tabular}{|c|c|c|c|c|c|}
\hline & & Enfermeiro (f) & Técnico (f) & Total (f) & $\%$ \\
\hline \multirow[t]{2}{*}{ Gênero } & Masculino & - & 5 & 5 & 13 \\
\hline & Feminino & 8 & 25 & 33 & 87 \\
\hline \multirow[t]{4}{*}{ Faixa etária } & 21 a 30 anos & 5 & 9 & 14 & 37 \\
\hline & 31 a 40 anos & 2 & 8 & 10 & 26 \\
\hline & 41 a 50 anos & - & 7 & 7 & 18 \\
\hline & 51 a 60 anos & 1 & 6 & 7 & 19 \\
\hline \multirow[t]{5}{*}{ Escolaridade } & $\begin{array}{l}\text { Fundamental } \\
\text { completo }\end{array}$ & - & 3 & 3 & 8 \\
\hline & Médio completo & - & 17 & 17 & 45 \\
\hline & Superior incompleto & - & 6 & 6 & 16 \\
\hline & Superior completo & 2 & 3 & 5 & 13 \\
\hline & Pós-graduaçãa & 6 & 1 & 7 & 18 \\
\hline \multirow[t]{3}{*}{ Renda individual * } & < 1 salário mínimo & - & 3 & 3 & 8 \\
\hline & 1 a 3 salários mínimos & 2 & 19 & 21 & 55 \\
\hline & > 3 salários mínimos & 6 & 8 & 14 & 37 \\
\hline \multirow[t]{5}{*}{ Estado civil } & Solteiro & 6 & 2 & 17 & 45 \\
\hline & Casado & 2 & 1 & 12 & 31 \\
\hline & Divorciado & - & 2 & 2 & 5 \\
\hline & Viúvo & - & 1 & 1 & 3 \\
\hline & $\begin{array}{l}\text { União } \\
\text { estável/amasiado }\end{array}$ & - & 6 & 6 & 16 \\
\hline \multirow[t]{5}{*}{ Religião } & Católico & 2 & 12 & 14 & 37 \\
\hline & Protestante & 3 & 5 & 8 & 21 \\
\hline & Espírita & 1 & 7 & 8 & 21 \\
\hline & Sem religião & 1 & 2 & 3 & 8 \\
\hline & Outra & 1 & 4 & 5 & 13 \\
\hline
\end{tabular}

* O salário-mínimo correspondia a $\mathrm{R} \$ 724,00$

Quanto a capacidade dos profissionais de enfermagem em auxiliar um paciente pós-tentativa de suicídio, o estudo revelou que $61 \%$ dos profissionais responderam ser capazes de prestar essa assistência, enquanto que 39\% negaram possuir essa capacidade, justificada em sua totalidade pela falta de preparo profissional.

Não porque, nós não tivemos treinamento e esta área é muito delicada. (T4) Se tivesse mais qualificação, sim. (T18)

Bom, não tenho nenhum treinamento específico então acho que não me sinto preparada. (T26)

A faculdade de enfermagem não prepara o profissional para lidar com esse tipo de assunto. (E8) 
No questionamento sobre a relação entre comportamento suicida associado a uma doença mental previa, 61\% dos profissionais de enfermagem relataram que esta relação é verdadeira e está fortemente presente nesses casos.

[...] distúrbios psicossociais, abuso de drogas lícitas e ilícitas, histórico de abusos. (E2)

Depressão [...]. (T1)

Eu penso que o indivíduo que tenta o suicídio deve-se a [...] distúrbios da saúde mental e outros. (T5)

Distúrbio mental. (T22)

Quanto a identificação dos fatores de risco para o suicídio na percepção dos profissionais da enfermagem, 71\% declararam que seriam capazes de fazer essa identificação.

Doenças terminais - Doenças sem perspectiva de melhora -Depressão. (E1)

Mudança comportamental - tentativa de pegar objetos q sirvam como arma. (E4)

Sim. Através da comunicação. (E7)

[...] às vezes pouco comunicativo, apático. (E8)

Primeiro lugar a depressão, depois os problemas familiares e por fim a estrutura financeira. (T14)

Sim mudanças de comportamento. Se apegam em objetos que poderiam usar como arma. (T29)

Quanto aos sentimentos dos profissionais de enfermagem durante a abordagem de paciente pós-tentativa de suicídio, $24 \%$ se sentem desconfortáveis em ofertar esse cuidado e $24 \%$ se mostram indiferentes ao prestar essa assistência.

Sobre o pensamento, a respeito dos pacientes atendidos após tentarem suicídio, comparados aos demais pacientes internados, percebeu-se que a maioria dos profissionais reconhecem a mesma ou maior importância no atendimento desse público.

Eu penso que ela está doente também, mas doente mental. Precisa de ajuda. (T5)

Eu acho que se uma pessoa que quer tentar suicídio entrar em um hospital, olhar para um paciente que está lutando pela vida. Ele vai dar valor em sua própria vida. (T16) 
Paciente que tem esse tipo de comportamento, merece mais atenção $e$ tratamento tanto quantos os outros, pois acredito que seja uma doença também. (T18)

Não dá para comparar, todos nós somos indivíduos e devemos ser tratados como tal; cada um enxerga a vida por uma ótica; a vivência, experiência, o que motiva a vida de cada um deve ser considerada; são situações diferentes. (E6)

Com relação a autonomia do sujeito em atentar contra sua própria vida, verificou-se que os profissionais acreditam em uma forma de liberdade, desde que não envolva a vida de outros.

Depende da situação de cada um, existem tratamentos que no início da doença e expectativa de melhora do próprio indivíduo é possível. (T2)

Desde que não envolva terceiros. (T9)

Sim, desde que não envolva outras pessoas. (T17)

O estudo revelou ainda, que a maioria dos profissionais saberiam orientar os pacientes e familiares sob os cuidados posteriores a alta hospitalar, principalmente envolvendo outros profissionais especializados nessas demandas.

O ato do suicídio é apenas o estopim de um problema muito maior; tento orientar (...)digo para procurar ajuda psicológica e fala palavras de apoio. (E6)

Sim, a orientação se o indivíduo ainda não passou por profissionais de saúde mental, orienta a procurar um CAPS I de transtornos mentais, lá ela vai passar pelo psicólogo(a), psiquiatra(a), equipe de enfermagem capacitada e treinada para dar continuidade ao seu tratamento. (T11)

Sim, orientando na busca por ajuda psicológica para o indivíduo e seus familiares, sobre a importância de uma crença religiosa como base de apoio a todos os envolvidos, e o diálogo ou a busca pelo diálogo e compreensão das razões que levaram o indivíduo a este ato, para que a família possa compreender as dificuldades, o sofrimento, a causa, motivo que culminou com a tentativa do suicídio. (T12)

\section{DISCUSSÃO}

Estudos apontam que historicamente e culturalmente a mulher tem ocupado funções relacionadas com o cuidado ${ }^{7}$, corroborando com a maior prevalência entre os profissionais do gênero feminino verificados neste estudo.

Quanto a escolaridade dos profissionais, predominou ensino médio completo, com rendimentos mensais de 1 a 3 salários mínimos. De acordo com o IBGE, o salário está diretamente proporcional ao nível de escolaridade e qualificação do trabalhador ${ }^{8}$. 
Observa-se, no entanto uma porcentagem considerável de profissionais técnicos informando estarem no nível superior (completo e incompleto) correspondendo ao novo quadro e perfil de profissionais do país?.

0 estado conjugal predominante foi de solteiros, seguido de casados. Resultados também encontrados em estudo realizado com enfermeiros na atenção básica (36,7\% e $49,4 \%$ respectivamente) ${ }^{10}$. Prevaleceu também profissionais católicos, dado este que confirma o catolicismo como a religião majoritária, mas vem apresentando queda de adeptos desde a década 90, e o aumento dos protestantes ${ }^{11}$.

Apesar de maior parte dos profissionais explicitarem capacidade em auxiliar o paciente pós-tentativa de suicídio, chama atenção a justificativa daqueles que negaram essa capacidade. Em estudo com profissionais de enfermagem que atuam em um setor emergencial, observou-se o reconhecimento a respeito do pouco preparo na abordagem a paciente com emergência psiquiátrica bem como um conhecimento quase popular e baseado em experiências de outros no trato de tal paciente ${ }^{12}$. Esse panorama, demonstra a necessidade de inserir cotidianamente a temática do sofrimento mental e da subjetividade na prática de enfermagem, quer seja no momento de formação ou durante a assistência profissional, a fim de prestigiar cuidados sensíveis e humanizados ${ }^{12}$.

Atrelado às possíveis causas que poderiam estar associadas ao comportamento suicida neste estudo os mais apontados foram: os transtornos mentais, a ausência de religiosidade; história de vida; conflito familiar; situação emocional com carga negativa de estresse; relacionamento afetivo desastroso e condição financeira. Em específico, como um fator desencadeante do comportamento suicida, citaram fatores genéticos; covardia frente à vida; desocupação social e abandono de um programa terapêutico.

Em pesquisa sobre a prevalência de transtornos mentais em casos atendidos de tentativa de suicídio, observou que a $71,9 \%$ apresentavam transtornos mentais, dado que relacionado com os resultados de estudos de outros países, mostrando taxas semelhantes e até maiores, chegando a 90\%, como na Europa e Estados Unidos da América ${ }^{13}$.

Estudos apontam que há uma relação bem estabelecida entre o suicídio e os diversos transtornos mentais, sendo a depressão o transtorno psiquiátrico com maior fator de risco ${ }^{14}$.

No que concerne a identificação dos fatores de risco para o suicídio na percepção dos profissionais da enfermagem, os profissionais buscam no comportamento 
algum indício que leve a suspeita de tentativa de suicídio, seja na fala da ideação suicida ou no silêncio ${ }^{15}$. Em estudo onde entrevistou familiares de pessoas que tentaram suicídio ou suicidaram-se, revelou-se que a mudança de comportamento foi observada nas vítimas, identificando especialmente o isolamento e a tristeza ${ }^{16}$.

O fator de risco do ambiente e das condições demográficas presentes nas narrativas, corresponde com o encontrado na literatura, acrescentando a esses fatores, as situações clínicas mórbidas, alterações da imagem pessoal e o acesso facilitado a meios letais ${ }^{17}$. Em estudo de autópsia psicológica baseado em bilhetes suicidas, foi demonstrado que questões financeiras do indivíduo podem fazer parte dos fatores de um comportamento pré-suicida ${ }^{18}$.

Autores informam que alguns profissionais de saúde não estão preparados para detectar componentes suicidas, nem para aceitar o paciente suicida como uma pessoa que necessita de ajuda, permanecendo com posturas preconceituosas e discriminadoras em relação a esse tipo de paciente ${ }^{14}$.

Os sentimentos de julgamento e indiferença foram facilmente observados entre esses profissionais. Algo semelhante também foi encontrado em estudo que registrou como os profissionais viam o ato do suicídio, indicando como sendo um fato que ocorre em um momento de desespero ou uma forma de chamar atenção do ambiente ao redor, o que thes despertava diversos sentimentos, desde a caridade ao desprezo ${ }^{15}$.

Observou-se que a grande maioria das orientações direcionava a busca de uma religião e no geral, estavam associadas à busca por profissionais de saúde mental. Ademais, esses profissionais percebem a importância da família no suporte emocional do indivíduo e como ela se desestrutura frente a um evento suicida, porém sentem-se incapazes de fornecer um apoio emocional ${ }^{19}$.

A família é um elemento importante que auxilia os profissionais de saúde em seu cuidado, pois essa aproximação familiar promove a desmistificação do suicídio e seu comportamento e melhor manejo na prevenção de futuras tentativas ${ }^{20}$, estratégia essa preconizada pela Política Nacional da Saúde Mental, através da rede de Centros de Atenção Psicossocial (CAPS), em seu artigo $2^{\circ}$, parágrafo único inciso II, garante 0 alcançar da sua recuperação pela inserção na sua família e na comunidade, demonstrando a importância de ser realizado atividades de educação permanente entre os profissionais e se possível envolver a família nessa discussão, a fim que todos possam compreender o ato suicida e tornar-se um aliado no tratamento ${ }^{21}$. 


\section{CONSIDERAÇÕES FINAIS}

Verificou-se no estudo que a equipe de enfermagem percebe que o indivíduo que tenta suicídio, frequentemente está sofrendo de transtornos mentais, marcados por falta de estrutura familiar, dificuldades financeiras, problemas de relacionamento, ausência de religiosidade e estresse.

Esses profissionais demonstram ser capazes de identificar os fatores ou situações de risco ao suicídio, tanto naqueles inseridos na ambiência hospitalar quanto nos demais sujeitos, no convívio comunitário. E, ainda que haja facilitada identificação desses vulneráveis, os profissionais apresentam comportamentos distintos ao esperado por um cuidador, sinalizando para um processo de incompreensão ou negligência do sofrimento do outro.

Os resultados apontam para necessidade de novas e inovadoras formas de aprendizado para esse grupo de profissionais, enxergando na implementação do programa de educação permanente e comunicação horizontalizada entre as categorias de enfermagem, os primeiros passos para mudança desse cenário. Entretanto, por entender que o cuidador para assistir seu cliente deve possuir condições de saúde favoráveis, essa condição deve ser priorizada antes de assumir qualquer assistência.

\section{REFERÊNCIAS}

1. DeLeo D, Bertolote J, Lester D. Self-directed violence. In: Krug EG, Dahlberg LL, Mercy, JA editors. World report on violence and health. Geneva: World Health Organization; 2002. [acesso em 2016 abr. 12]. Disponível em: http://apps.who.int/iris/bitstream/10665/42495/1/9241545615_eng.pdf.

2. Associação Brasileira de Psiquiatria (ABP). Comportamento suicida: conhecer para prevenir (dirigido para profissionais de Imprensa). Rio de Janeiro (RJ): ABP editora; 2009.

3. Brasil. DATASUS - Departamento de Informática do SUS / Ministério da Saúde. Indicadores de mortalidade. Óbitos por suicídio por unidade de federação segundo região. Brasilia: 2011 [acesso em 2016 abr. 12]. Disponível em: http://tabnet.datasus.gov.br/cgi/tabcgi.exe?idb2012/c09.def. 
4. Avanci RC, Furegato ARF, Scatena MCM, Pedrão LJ. Relação de ajuda enfermeiropaciente pós-tentativa de suicídio. SMAD, Rev eletrônica saúde mental alcool drog. 2009;05(1):1-15.

5. Waiselfisz JJ. Mapa da violência 2014: os jovens do Brasil. Rio de Janeiro: UNESCO; 2014. [acesso em 2015 set. 15]. Disponível em: http://www.mapadaviolencia.org.br/pdf2014/Mapa2014_JovensBrasil_Preliminar. pdf

6. Minayo MCS. O desafio do conhecimento: pesquisa qualitativa em saúde. 9th ed. São Paulo: Hucitec; 2006.

7. Harbs TC, Rodrigues ST, Quadros VAS. Estresse da equipe de enfermagem de um centro de urgência e emergência. Bol enferm. 2008;1(2):41-50.

8. Instituto Brasileiro de Geografia e Estatística - IBGE (BR). Principais destaques da evolução do mercado de trabalho nas regiões metropolitanas abrangidas pela pesquisa: Recife, Belo Horizonte, Rio de Janeiro, São Paulo e Porto Alegre - 20032013. Rio de Janeiro; 2013 [acesso em 2014 set. 15]. Disponível em: http://www.ibge.gov.br/home/estatistica/indicadores/trabalhoerendimento/pme nova/retrospectiva2003_2013.pdf.

9. Instituto Brasileiro de Geografia e Estatística - IBGE (BR). Síntese de indicadores sociais - uma análise das condições de vida da população brasileira. Rio de Janeiro; 2013 [acesso em 2015 jan. 10]. Disponível em: http://biblioteca.ibge.gov.br/visualizacao/livros/liv66777.pdf.

10. Corrêa ACP, Araújo EF, Ribeiro AC, Pedrosa ICF. Perfil sociodemográfico e profissional dos enfermeiros da atenção básica à saúde de Cuiabá - Mato Grosso. Rev eletrônica enferm. 2012; 14(1):171-180.

11. Instituto Brasileiro de Geografia e Estatística - IBGE (BR). Censo 2010. Número de católicos cai e aumenta o de evangélicos, espíritas e sem religião. Rio de Janeiro; 2010. [acesso em 2016 abr. 13]. Disponível em: http://censo2010.ibge.gov.br/noticias-

censo. html?view=noticia\&id=3\&idnoticia $=2170 \&$ busca $=1 \& \mathrm{t}=$ censo-2010-numerocatolicos-cai-aumenta-evangelicos-espiritas-sem-religiao.

12. Kondo EH, Vilella JC, Borba LO, Paes MR, Maftum MA. Abordagem da equipe de enfermagem ao usuário na emergência em saúde mental em um pronto atendimento. Rev esc enferm USP. 2011; 45(2):501-507. 
13. Santos AS, Lovisi G, Legay L, Abelha L. Prevalência de transtornos mentais nas tentativas de suicídio em um hospital de emergência no Rio de Janeiro, Brasil. Cad saúde pública. 2009; 25(9):2064-2074.

14. Carvalho MB. Psiquiatria para a enfermagem. São Paulo (SP): Rideel; 2012.

15. Silva VP, Boemer MR. O suicídio em seu mostrar-se a profissionais de saúde. Rev eletrônica enferm. 2004; 6(2):143-52.

16. Pordeus AMJ, Cavalcanti LPG, Vieira LJES, Coriolano LS, Osório MM, Ponte MSR, et al. Tentativas e óbitos por suicídio no município de Independência, Ceará, Brasil. Ciênc saúde coletiva. 2009; 14(5):1731-40.

17. Botega NJ, Werlang BSG, Cais CFS, Macedo MMK. Prevenção do comportamento suicida. Psico (Porto Alegre). 2006; 37(3): 213-20.

18. Kovács MJ. Morte e desenvolvimento humano. 5th ed. São Paulo: Casa dos Psicólogos; 1992.

19. Buriola AA, Arnauts I, Decesaro MS, Oliveira MLF, Marcon SS. Assistência de enfermagem às famílias de indivíduos que tentaram suicídio. Esc Anna Nery Rev. Enferm. 2011; 15(4):710-16.

20. Heck RM, Kantorski LP, Borges AM, Lopes CV, Santos MC, Pinho LB. Ação dos profissionais de um centro de atenção psicossocial diante de usuários com tentativa e risco de suicídio. Texto \& contexto enferm. 2012; 21(1):28-33.

21. Brasil. Lei 10.216, de 06 de abril de 2001. Dispõe sobre a proteção e os direitos das pessoas portadoras de transtornos mentais e redireciona o modelo assistencial em saúde mental. Diário Oficial da União. Brasília, 6 de abril de 2001. [acesso em 2016 abr. 13]. Disponível em: http://www.planalto.gov.br/ccivil_03/leis/LEIS_2001/L10216.htm.

Conflito de interesses: Os autores declaram não haver conflito de interesses.

Como citar este artigo: Liba YHAO, Lemes AG, Oliveira PR, Nascimento VF, Fonseca PIMN, Volpato RJ, et al. Percepções dos profissionais de enfermagem sobre o paciente pós-tentativa de suicídio. Journal Health NPEPS. 2016; 1(1):109-121. 